\title{
Individual and family issues surrounding a rare disease
}

\author{
Susan Ann Vitale* \\ School of Nursing, Molloy College, Rockville Centre, NY, United States
}

Received: July 26, 2017

Accepted: October 15, 2017

Online Published: October 27, 2017

DOI: $10.5430 / \mathrm{cns} . v 6 \mathrm{n} 1 \mathrm{p} 45$

URL: https://doi.org/10.5430/cns.v6n1p45

\begin{abstract}
Objective: The purpose of this research was to gain an understanding of individual and family experiences of living with a rare disease.

Background: There are over 7,000 types of rare diseases affecting $>25$ million individuals in the United States (US) and more worldwide. Individuals with a rare disease are geographically separated and may encounter care providers unfamiliar with their disease. They often face diagnostic delays, inadequate treatment options or a lack of hope for a cure. Economic and societal implications include high costs associated with morbidity and mortality. While there is some research on individual rare disease experiences, a review of experiences common to a wide variety of rare diseases for potential commonalities has not been established.

Methods: A qualitative descriptive design of case study methodology involved data collection through individual and small group interviews and also by email. Analysis identified themes and commonalities found within the rare diseases represented in this study.

Results: Thirty-five adults diagnosed with a rare disease and some family members participated. Data revealed phases of adjustment and themes associated with those experiences. Themes included isolation or feeling all alone, frustrations, searching for a specialist, developing disease expertise, finding outside support, striving for stability and normalacy and maintaining hope. Conclusions: The participants in this study faced their rare disease obstacless with courage. Appreciation of their varied disease experiences contributes to a fund of limited information on rare disease experiences.
\end{abstract}

Key Words: Rare disease/disorder, Qualitative, Individual/family experiences

\section{INTRODUCTION}

The purpose of this research was to gain an understanding of the experience of individuals and families when they are living with a rare disease. Little research exists on various rare disease experiences in both adult and pediatric populations. Those providing guidance to patients or to their families do not have the benefit of a well-studied understanding of commonalities that exist across various types of rare diseases.

\section{BACKGROUND OVERVIEW OF RARE DIS- EASES}

There are more than 7,000 rare disorders affecting an estimated 25-30 million individuals in the US as well as millions more throughout the world. A disease in the US is considered rare if it has a prevalence of fewer than 200,000 affected individuals at any one time. ${ }^{[1]}$ Rare diseases are often complex and may involve a chronic illness, disability, or cause premature death. They also occur in children and may have States.

*Correspondence: Susan Ann Vitale, Prof.; Email: svitale@ molloy.edu; Address: School of Nursing, Molloy College, Rockville Centre, NY, United 
a genetic origin. While individual disease incidence is small, the collective number of all types of rare diseases is large. Available literature and research on these uncommon diseases is limited and in some cases non-existent. Individuals diagnosed with a rare disease and their families often face unusual health care issues, diagnostic delays, and inadequate treatment options. Their problems can be complicated by a lack of disease specific information and limited access to health care. Geographic separation from others with the same health challenges impedes interaction with or benefit from those who may have learned how to cope with disease consequences. ${ }^{[1,2]}$

\subsection{Limited research}

Rare diseases may recieve minimal attention from medical researchers as participant testing groups may lack adequate numbers. The US Orphan Drug Act of 1983 provided pharmaceutical company incentives for rare disease drug development. A few hundred drugs have been developed since then. Another effort is the Therapeutics for Rare and Neglected Diseases (TRND) program. It provides a coordinated effort for drug therapy development with a laboratory run by the US National Human Genome Research Institute. ${ }^{[3]}$ Such legislation provides incentives to stimulate research however it does not assure that research will occur leaving most with rare diseases without hope for treatment.

There is no wide scale inclusive database with which to track the incidence and treatment of all of the more than 7,000 known types of rare diseases. Some diseases are so rare that only a few people are affected, leaving causes or remedies unknown. Others have a potential treatment that remains unavailable or lacks financial incentives for development. While some progress has been made in prevention and therapy for certain rare diseases, the vast majority lack a cure or threatment. ${ }^{[3]}$

\subsection{Economic costs}

The etiology, cure, and treatment of rare diseases have economic consequences. Rare disease costs may be high for both patient and society when morbidity and disability are attributed to a lack of treatment. There are limited cost of illness studies that have been conducted on rare diseases. The cost of a rare disease involves both direct and indirect costs that may extend through a lifetime. Indirect costs may be associated with loss of the patient's economic productivity and may also include a family caregiver's loss of productivity. Rare diseases are clearly associated with significant economic burdens on both the involved individuals and society. ${ }^{[4]}$

\subsection{Benefits of the Internet}

Fortunately the rise in popularity of the Internet has helped to disseminate what limited knowledge may be available. It can help to build an online community for afflicted patients, frustrated with a lack of rare disease assistance. Not all rare diseases have an Internet support group in place. Individuals may turn to reputable Internet sources for rare disease information (see Table 1). The number of health information seekers for all diseases is increasing. People may tend to turn to the Internet for disease-specific information, review of their symptoms and search for assistance in seeking medical care. ${ }^{[5]}$ While there are few studies of Internet use related to rare diseases, it appears that the Internet can offer basic rare disease information, the means of locating a disease specialist, if one exists, and information on clinical drug trials. ${ }^{[6]}$

\subsection{Purpose of this study}

While there is some research on individual rare disease experiences, a review of a wide variety of rare disease experiences for potential commonalities amoung them all has not been established. This study focused on a variety of rare diseases in an attempt to understand the influence that rarity has on the personal reality and socially constructed nature of those common experiences to rare diseases in general. The lack of research on the experience of living with a rare disease leaves health care providers without adequate informational resources. The objective of this research was to explore the experiences of individuals diagnosed with a rare disease and immediate family members living with someone diagnosed with a rare disease. Family members were sought to provide the experience of parents or spouces caring for a child or an adult with a rare disease.

\section{RESEARCH METHOD AND PROCEDURES}

A qualitative descriptive design of case study methodology was used. Case study method ${ }^{[7]}$ is indicated when there is a clear focus on gaining an in-depth understanding of the actions of individuals with a common challenge, problem, or issue. The common challenge here was having a rare disease. In-person interviews were conducted individually and in small groups. Data was also gathered by email to offer convenience to participants who were geographically scattered due to the rare incidence of the disease or when individuals were too sickly to attend an in-person interview. Participants were asked a few broad open-ended questions with probes to help them tell their stories. They were asked to focus on their experiences with their rare disease, their challenges, concerns, and also their successes and support.

A constant comparative approach helped to inform the anal- 
ysis of identified themes and commonalities in the data. ${ }^{[8]}$ Researchers can use a variety of approaches for analysis. Therefore this approach was modified so as to begin a search through the data for broad categories and themes that would bring meaning to the experiences of the participants but not necessarily end with a full grounded theory. The coded data were searched for commonalities or similarities. This step was followed by a search for patterns or themes related to the disease experience.

Participants were informed of the study by a flyer and also recruited by "word-of-mouth". Thirty-five participants were interviewed. All were at least 18 years of age or older and fluent in English. They were adults with a diagnosis of a rare disease or an adult immediate family member of someone with a rare disease, such as a parent of a child. They provided basic demographic data and described symptoms, diagnostic steps, treatment, efficacy, and side effects. Interviews were open and unstructured to encourage personal stories to unfold. A personal journal and contextual field notes in- cluded the researcher's impressions of thematic aspects of the data. The transcribed data were read and re-read many times over to search for.a rich description of the phenomenon of a having rare disease. Themes were extrapolated from the participants' descriptions. Ongoing analysis began during data collection. Themes of special concern were identified and examined for commonalities and differences between participants. ${ }^{[9]}$

\section{Ethical standards}

This research was approved by a college institutional review board for the protection of human subjects. Confidentiality of the participants was protected. Identifying information, such as residential or work location was deleted from transcribed data. Integrity and quality of the research were evaluated through collegial support, journaling and an audit trail for veracity of expressed meanings and reliability. Trustworthiness was established through participant agreement with the findings.

Table 1. Demographics

\begin{tabular}{ll}
\hline Variety & Patient themselves = 19 \\
\hline \multirow{2}{*}{ Participants = 35} & Mothers of a patient =14 \\
& Fathers of a patient = \\
Participant Gender & $\mathrm{M}=11 / \mathrm{F}=24$ \\
Patient Age Range & $2-69$ years \\
Marital Status & Married/living with a significant other = 23 \\
& Widowed = 2; Divorced/Separated = 5 unmarried = 5 \\
& Ehlers Danlos Syndrome, Scleroderma, Addisons/Poly Glandular Failure, Evans Syndrome, Klippel-Fiel \\
& Syndrome, Russell Silver Syndrome, Mycosis Fungoides, Cutaneous T-Cell Lymphoma, Aplastic \\
& Anemia, Olivopontocerebellar Atrophy, Pseudotumor Cerebri, Myasthenia Gravis, Dyskeratosis, \\
Rare Diseases & Pseudo-Hypoaldosteronism, Parry-Romberg Syndrome, Reflex Sympathetic Dystrophy, Prader-Willi \\
Represented & Syndrome, Charcot-Marie Tooth, Ichthyosis, Klippel-Trenaunay, Niemann Pick Disease, Sturge-Weber \\
& Syndrome, Angelman Syndrome, Pseudo Obstruction, Agenesis of the Corpus Callosum, Growth \\
& Hormone Deficiency, Stiff Person Syndrome (2), Hereditary Spastic Paraplegia (2), Primary Lateral \\
& Sclerosis (2), Epidermolysis Bullosa (3) \\
&
\end{tabular}

\section{STUDY FINDINGS}

The demographic characteristics of the 35 participants were varied. There were 19 adults reporting on their own rare diseases. The remaining 16 participants were parents (14 mothers/2 fathers) reported on their children of various ages and diagnosed with a rare disease. Race and ethnicity was an optional data collection question and insufficiently collected. The majority of participants reported being married or living with a significant other who offered support. The ages of the individuals with rare diseases ranged from 2 to 69 . Their geographic locations varied, being rural, suburban and urban. Areas of the US included California, Connecticut, Florida,
Georgia, Iowa, Massachusetts, Michigan, New Jersey, New York, Oregon, South Carolina, Texas, Utah and Vermont. Two individuals communicated by internet from Canada and Italy. All hoped for but lacked a cure. Treatment ranged from none available to assorted types with a wide range in efficacy and side effects. Their rare diseases were varied and included some that were of genetic origin (see Table 1).

Phases of Adjustment There were three overriding phases of adjustment to living with a rare disease (see Figure 1). The phases of adjustment began with the first phase of "facing a rare disease diagnosis". Each individual had to begin by confronting the rare disease diagnosis. This was often 
a shock or total surprise for both the individual with the diagnosis and the family or significant other. None expected this. A common expression was "why me" or "what did I do to deserve this". One mother said "this hit me really hard," another parent said "I felt like I just got shot.... its my kid, my child, its unbelievable." The next phase was identifying and participating in treatment specific to the rare disease. However for some this meant finding that there was a lack of treatment for the disease. The third phase was learning how to cope with rare disease issues. The participants cycled through the phases or even repeated the phases as the disease progressed or exacerbated or if they reached a stage of remission or treatment efficacy. There was not a unpredictable or stable cycle of phases of adjustment.

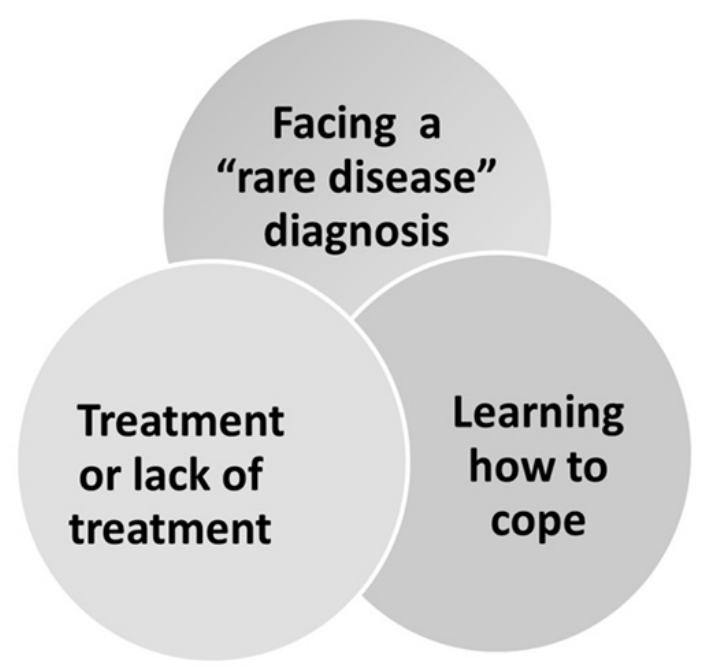

Figure 1. Phases of a rare disease experience

Themes were identified that were commonly found through all of the three phases of adjustment (facing a rare disease, treatment or lack of treatment, and learning how to cope). The themes were also found in all types of rare diseases that the participants were living with (see Table 2). Varying levels of emotional responses were associated with their experiences. For some a feeling, such as isolation, was intense. For others it was present but less bothersome, especially if social connections were found through the Internet or within a disease related support group. Frustration was intermittent and often related to disease exacerbations. The identified themes are described as follows.

Table 2. Themes

\begin{tabular}{ll}
\hline Emotional responses & \\
\hline Isolation: Feeling all Alone & Finding Outside Support \\
Frustrations & Striving for Stability and Normalacy \\
Searching for a Specialist & Maintaining Hope \\
Developing Disease Expertise & \\
\hline
\end{tabular}

Isolation: Feeling all alone: Initially many described feeling stunned and desperate upon learning of the diagnosis. Individuals felt all alone and isolated from others with a like disease. Some described feeling unusual in knowing their disease was rare. If there were obvious signs of the illness such as a skin condition or disabiling physical appearance they felt "unattractive" or even stigmatized. While the Internet was helpful, one young man with Charcot-Marie Tooth said it was his only connection to others with his condition. However, he also indicated the information he found was "scarey". He had hoped to work as a pilot but realized that was not going to be an option for him.

Frustrations: Frustrations were felt when treatment was ineffective, unavailable or even non-existent. Anger, deression, and sadness was intermingled with hope and periods of happiness. Individuals knew the situation was unique and rare with many challenges that others were not aware of. They spoke of feeling "ups and downs" along their disease trajectory. A man with olivo-ponto cerebellar degeneration said "no one really understands what it is like.... there is no cure and so I just have to live with this awful thing. My brain and spine aren't working well... they tell me it is degeneration, meaning slowly getting worse. I have trouble walking and getting around".

Searching for a specialist: Referrals were made by primary care but often the patient or family member identified a specialist themselves through the Internet. Most individuals began an immediate search for disease information and specialists themselves. Unfortunately some could not be located or easily reached if at a distance from their home. If a disease was quite rare a specialist could be thousands of miles away. Not all had resources for such travel. One participant was aware of how fortunate she was to live in an urban area with major medical centers near by. Her care in a cutaneous T-Cell lymphoma clinic was right within her neighborhood. She spoke of others traveling great distances to attend that same clinic.

Developing disease expertise: The Internet became the main source of disease information and support. Information searches helped give a sense of control over a disease that was often out of control, untreatable or uncurable. Knowledgeable involvement during doctor appointments felt empowering. Being a true partner in care decisions was valued by the participants. One said: "I need to be in charge....I'm the one who has this awful thing so I gotta know all about it..... I need to be involved with the doctors." Another mentioned that she could only really count on herself, so she tried to read everything she could find on her disease.

Finding outside support: They searched for medical help, 
educational materials and support people that understood what their unusual disease was about. Support from family and friends varied due to obligations that others were involved with. Online support was seen as dependable. It could provide disease information. An online support group was cherished as a lifeline, especially if others with the disease were located very far away. Three parents of children with epidermolysis bullosa all mentioned feeling devastated over the diagnosis but all felt highly supported by the Internet and the non-profit disease organization. One of them said "I could never do this alone (care for her daughter's blistering fragile skin). My child needs daily painful wound care... they teach us and even provide a medical help". A parent of another child with Prader Willi syndrome said the Internet and a national disease association were her "... lifeline... I just don't see how I would go about this and be a good parent ... I avoided connecting with the support group for a few years but I was a mess. I needed help and found relief with the other parents".

Striving for stability and normalcy: Individuals, especially parents had to perservere no matter how emotional they felt about the diagnosis. There was a need to adjust and take care of the sick family member. Parents tried to "push upset feelings back and act normal". Even the adults living with the disease would try this at times to help themselves and others feel better. The goal was to be a "good patient" or a "good family member". Some mentioned trying to look "nomal" or "not unusual" even if they did not feel that way. The positive effects this had on others rebounded back to them in an increase in socialization. One mentioned that she had to make sure visitors did not feel uncomfortable with her illness. Another said "people tend to not show up any more, even family. It is hard to be so alone and not be able to be social as I'd like... I look funny or scarey or something unusual."

Maintaining hope: Hope was maintained in the midst disease complications. It was difficult to be hopeful if disease effects were hard to endure. Sometimes hope was in the background in wishing for a cure. One described how he must "hold on" even if he was "loosing grip". He had to try very hard at times "to just live".

\section{DiscuSSION}

The phases of the disease experience and the themes that emerged from this study suggest that there are universal human experiences of living with a rare disease. The individuals and families faced and managed many impediments. They were disheartened by limited available disease information and resources to varying degrees. However, they maintained hope for a future eventual cure and effective treatment regarding themselves and others in similar situations.

\subsection{Study limitations}

The participants and families who shared their stories may have been an unusual group and differ from a wider group of those with differing diseases, problems or resources to cope. The participants in this study were a convenience sample of all adults. Children were not interviewed and might yeild other information. Ethnicity and race were not reliabily identified and might have offered valuable information.

\subsection{Implications for practice}

The conclusions drawn from the journeys shared here can build a foundation from which to better understand the needs of individuals and their families who will encounter a rare disease in the future. The identified themes provide a focus for communication with newly-diagnosed patients and families as they respond to the anxiety of having a rare disease. Further development of rareness as a potential concept in illness would be integral to nursing care. Clearly defined attributes, empirical referents, and consequences of rareness in illness, remain undetected. Clinicians and nurse educators working with patients with a rare disease need to be cognizant of potential difficulties such as isolation, stigmatization, inadequate or misunderstood disease information, or treatment adherence (see Table 3). Information on rare diseases needs to be disseminated to increase public and health professional consciousness (see Table 4).

Table 3. Implications for practice

- Identify Community And Internet Resources

- Consider Referrals To Specialists

- Anticipate Potential Social Isolation

- Anticipate Frustrations Mixed With Hope

Table 4. Resources for patients and families

\begin{tabular}{ll}
\hline Resources & \\
\hline Genetic and Rare Diseases (GARD) Information Center. & $\underline{\text { http://rarediseases.info.nih.gov/GARD }}$ \\
National Organization for Rare Diseases, & $\underline{\text { http://www.rarediseases.org }}$ \\
Genetic Alliance & $\underline{\text { http://www.geneticalliance.org }}$ \\
\hline
\end{tabular}




\subsection{Implications for future research}

A research project of this scope could not encompass all aspects of the individuals and families contending with a rare disease. There is a need to further understand how individuals cope and maintain quality in their lives. Longitudinal qualitative research to better understand the complex factors of uneven disease trajectory, remissions, exacerbation of symptoms, and new treatments would need to be part of such a design. Such research should lead to providing timely interventions following diagnosis and the development of protocols for promoting health maintenance and adaptation to rare diseases. Rare disease research warrants a higher emphais so that the afflicted might be provided more comprehensive care and quality of life.

\section{Conclusions}

This study has been a step towards opening the pathway for a clearer professional appreciation of the needs, concerns, and adaptations of those diagnosed with a rare disease. It is hoped that these findings will provide the foundation for further investigation into the experience of living with rare diseases.

\section{CONFlicts OF InTEREST Disclosure}

The authors declare they have no conflicts of interest.

\section{REFERENCES}

[1] NORD (ND). National Organization for Rare Disorders: Rare Disease Information for Medical Professionals. July 1, 2017. Available from: http://nordphysicianguides.org/

[2] National Institute of Health. Rare Diseases. 2017. Available from: https://medlineplus.gov/rarediseases.html

[3] National Human Genome Research Institute. Frequently Asked Questions About Rare Diseases. 2012. Available from: https: //www.genome.gov/27531963/

[4] Angelis A, Tordrup D, Kanavos P. Socio-economic burden of rare diseases: A systematic review of cost of illness evidence. Health Policy. 2015; 119(7): 964-79. PMid: 25661982. https://doi .or $\mathrm{g} / 10.1016 / \mathrm{j}$. healthpol.2014.12.016

[5] Koch-Weser S, Bradshaw YS, Gualtieri L, et al. The Internet as a health information source: findings from the 2007 Health Informa- tion National Trends Survey and implications for health communication. Journal of Health Community. 2010; 15(Suppl 3): 279-293. https://doi.org/10.1080/10810730.2010.522700

[6] Morgan T, Schmidt J, Haakonsen C, et al. Using the Internet to Seek Information About Genetic and Rare Diseases: A Case Study Comparing Data From 2006 and 2011. JMIR Research Protocols. 2014; 3(1): e10. https://doi.org/10.2196/resprot. 2916

[7] Yin RY. Case Study Research: Design \& Method (5 ${ }^{\text {th }}$ Ed). Los Angeles CA: Sage; 2014.

[8] Miles MB, Huberman AM, Saldana J. Qualitative Data Analysis: A Methods Source Book ( $3^{r d}$ Ed) . Los Angeles, CA: Sage; 2013.

[9] Bazeley P. Qualitative Data Analysis: Practical Strategies. Los Angeles, CA: Sage; 2013. 